\title{
REVIEW
}

\section{Novel therapies in osteoporosis: PTH-related peptide analogs and inhibitors of sclerostin}

\author{
Tilman D Rachner1,2,3, Lorenz C Hofbauer1,2,3,4, Andy Göbel1,3 and Elena Tsourdi1,2,3 \\ 1Department of Medicine III, Technische Universität Dresden Medical Center, Dresden, Germany \\ ${ }^{2}$ Center for Healthy Aging, Technische Universität Dresden Medical Center, Dresden, Germany \\ ${ }^{3}$ German Cancer Consortium (DKTK), partner site Dresden and German Cancer Research Center (DKFZ), Dresden, Germany \\ ${ }^{4}$ Center for Regenerative Therapies Dresden, Technische Universität Dresden, Dresden, Germany
}

Correspondence should be addressed to T D Rachner: tilman.rachner@uniklinikum-dresden.de

\begin{abstract}
Bone-forming approaches to treat patients with severe osteoporosis are effective, but treatment options are limited, and there is an unmet clinical need for additional drugs. This review discusses two novel and advanced anabolic therapeutic concepts that have successfully completed phase 3 trials. Romosozumab is a monoclonal antibody that targets the Wnt inhibitor sclerostin. Two phase 3 trials (FRAME and ARCH) of romosozumab for the treatment of postmenopausal osteoporosis have been completed. Both trials successfully reached their primary endpoint by reducing vertebral fractures by $75 \%$ compared to placebo (FRAME trial) and 48\% compared to alendronate (ARCH trial), respectively. Abaloparatide is a PTH-related protein (PTHrP) analog that has displayed bone anabolic activity. In the phase 3 ACTIVE trial, abaloparatide was compared to placebo and teriparatide for 18 months in postmenopausal women who had already experienced an osteoporotic fracture. Abaloparatide successfully reduced the rate of new vertebral fractures by $86 \%$ compared to placebo. Furthermore, abaloparatide achieved greater BMD increases at all measured sites compared to both placebo and teriparatide. Based on these results, abaloparatide was FDA approved in April 2017. This review discusses available data of both agents with regard to efficacy and safety as well as their possible future application.
\end{abstract}

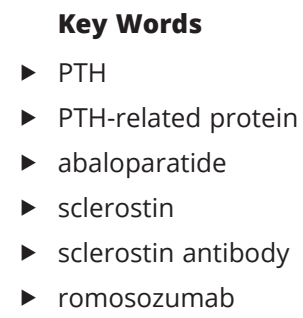

Journal of Molecular Endocrinology (2019) 62, R145-R154

\section{Introduction}

Antiresorptive drugs constitute the mainstay of osteoporosis treatment to date. In particular, bisphosphonates have been the most commonly used drugs, with a proven efficacy to reduce the risk of vertebral, hip and non-vertebral fractures (Papapoulos 2011). Denosumab, a monoclonal antibody against the receptor activator of nuclear factor $\kappa \mathrm{B}$ ligand (RANKL), is another potent antiresorptive agent, which results in continuous bone mineral density (BMD) accrual, suppression of bone turnover markers and fracture risk reductions, while maintaining an acceptable safety profile for up to 10 consecutive years of treatment (Bone et al. 2017). Additional concepts like hormone replacement therapy or selective estrogen receptor modulators are available but generally restricted to women with less severe osteoporosis shortly after menopause.

These antiresorptive drugs suppress osteoclastogenesis and osteoclast function, resulting in suppression of bone turnover and thereby increase mineralization. However, the potency of this approach has limitations 
and structural defects are not corrected (Toulis et al. 2011). Some patients with severe forms of osteoporosis will require more aggressive approaches to prevent the occurrence of fractures.

For these patients, teriparatide, a parathyroid hormone (PTH) analog, was the first osteoanabolic drug to be approved for the treatment of osteoporosis. However, its application is only approved for up to 24 months and requires daily subcutaneous injections. Due to a lack of competition, teriparatide is considerably more expensive than any antiresorptive approach, further limiting its use to a small number of osteoporotic patients. With a clear unmet need for additional bone anabolic drugs, this review will discuss two advanced new osteoanabolic concepts; namely parathyroid hormone-related peptide (PTHrP) analogs and sclerostin inhibition.

\section{PTH and PTH-related peptide in bone}

The actions of PTH on bone are mediated through the parathyroid hormone 1 receptor (PTH1R), a G-proteincoupled receptor, which is expressed on osteoblasts and osteocytes (McCudden et al. 2005). A chronic sustained activation of the receptor by $\mathrm{PTH}$, as is the case in primary hyperparathyroidism, exerts catabolic effects on bone and leads to an enhanced bone turnover, resulting in bone loss and an increased fracture risk (Lewiecki \& Miller 2013) (Fig. 1). On the contrary, intermittent pulsatile exposure to PTH enhances bone formation (Frolik et al. 2003) and translates into increased bone mass and improved microarchitecture, particularly in the cancellous bone compartment (Leder 2017). Consequently, the approval of teriparatide, a PTH analog comprising the first 34 amino acids of the endogenous hormone, constituted an important advance in the treatment of osteoporosis. Teriparatide has proven efficacy in increasing the spine and hip BMD while reducing the risk of vertebral and non-vertebral fractures in the settings of postmenopausal osteoporosis, osteoporosis in men and glucocorticoidinduced osteoporosis (Leder 2017). One limitation in the mechanism of teriparatide is that while stimulating bone formation, a concomitant stimulation of bone resorption is observed, blunting the induced net bone gain (Glover et al. 2009).

While some of its actions overlap with PTH, PTHrP exerts a distinct role in certain aspects of bone biology
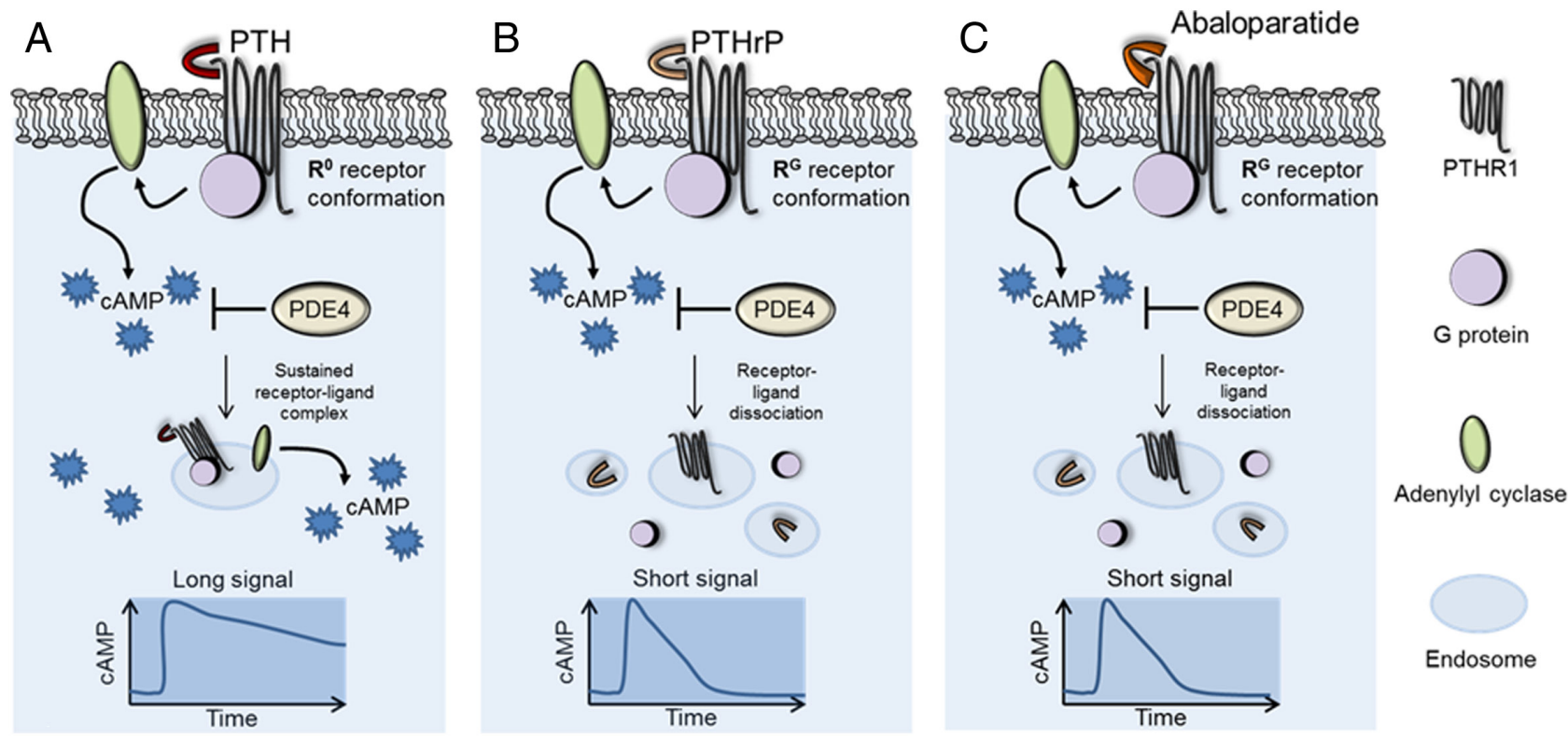

Figure 1

Abaloparatide is a synthetic peptide analog of parathyroid hormone-related peptide (PTHrP). Parathyroid hormone (PTH) activates the parathyroid hormone 1 receptor (PTHR1), a G-protein-coupled receptor on osteoblasts and osteocytes. Continuous presence of PTH favors bone resorption over formation by a sustained production of intracellular adenosine-3',5'-cyclic monophosphate (cAMP). Here, PTH is locked into the receptor and results in a stabilized $\mathrm{R}^{0}$ receptor conformation. Subsequent activation of adenylyl cyclase leads to a quick cAMP production right after peptide binding. The strong hormone-receptor complex is internalized into early endosomes and continuously produces cAMP leading to a sustained intracellular cAMP delivery. CAMP is degraded by phosphodiesterase-4 (PDE4) (A). Parathyroid hormone-related peptide (PTHrP) also functions through PTHR1 and induces the same quick CAMP production. By contrast to PTH signaling, PTHrP induces a short-signal RG receptor conformation and a more rapid and transient increase in CAMP. PTHR1 and the dissociated PTHrP are then degraded by endosomes at different locations. Short-cAMP-signaling is considered bone anabolic (B). Abaloparatide is a synthetic PTHrP analog which induces a similar signaling cascade thereby mediating anabolic signals in osteoblasts (C). 
and alterations in its functions are associated with various skeletal diseases. PTHrP is physiologically produced by mammary epithelial cells and is secreted into the milk and the systemic circulation. During lactation, circulating PTHrP activates bone resorption to mobilize maternal skeletal calcium stores that are required by the mammary gland for milk production (Wysolmerski 2012). PTHrP is best known as a mediator of cancer-induced humoral hypercalcemia (Wysolmerski 2012). High levels of circulating PTHrP then mediate an increased osteoclastic bone resorption, resulting in severe hypercalcemia.

PTH and PTHrP share eight of the first 13 amino acids and a similar secondary structure over the next 21 amino acids, and these homologous sequences allow them to bind and activate the same receptor PTH1R (Gensure et al. 2005). Recent research has revealed that there are two high-affinity PTHR1 conformations, namely the G-protein-independent $\mathrm{R}^{0}$ and the G-protein dependent $\mathrm{R}^{\mathrm{G}}$ conformation (Hattersley et al. 2016) (Fig. 1). Activation of the $\mathrm{R}^{0}$ conformation leads to a longer signaling activation than activation of the PTH1R in the $\mathrm{R}^{\mathrm{G}}$ conformation, which results in a rapid but very transient signaling response (Boyce et al. 2018). Based on these results, a more potent anabolic activation has

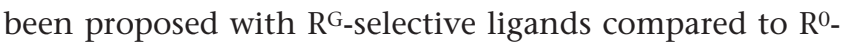
selective ligands (Cheloha et al. 2015).

\section{Abaloparatide}

Abaloparatide is a 34 amino acid synthetic analog of PTHrP, with a $41 \%$ homology to human PTH (1-34) and a $76 \%$ homology to human PTHrP (1-34), which binds transiently but with a greater selectivity to the $\mathrm{R}^{\mathrm{G}}$ conformation of PTHR1 on the surface of osteoblasts and osteocytes than teriparatide (Hattersley et al. 2016). This difference in the respective interaction with the PTHR1 is considered to mediate the distinctive clinical effects of these two drugs, with abaloparatide displaying more modest effects on bone resorption than teriparatide (Boyce et al. 2018). This is in concordance with previous human data showing that PTHrP administration causes a dosedependent increase in bone formation markers, while bone resorption markers remain virtually unchanged (Horwitz et al. 2010).

Preclinical studies in ovariectomized rats revealed that treatment with abaloparatide over 12 months led to enhanced bone formation in both trabecular and cortical compartments without concomitantly increasing bone resorption (Varela et al. 2017a). These effects translated into increased bone mass and bone strength in the spine and femur of these animals (Varela et al. 2017b). Subsequently, effects of treatment with abaloparatide were investigated using ovariectomized cynomolgus monkeys (cynos), which constitute a model considered to reflect many aspects of postmenopausal osteoporosis (Smith et al. 2009). Abaloparatide administration to ovariectomized cynos for up to 10 months induced BMD gains and preferentially increased bone formation as revealed by histomorphometry (Legrand et al. 2001). A recent study designed to evaluate longer-term effects over 16 months reproduced these findings, while also showing that abaloparatide treatment was associated with greater lumbar vertebral strength (Doyle et al. 2018).

Abaloparatide has recently been approved for the treatment of postmenopausal osteoporosis by the FDA. This decision was primarily based on the findings from a phase 2 dose-finding study (Leder et al. 2015) and from the Abaloparatide Comparator Trial in Vertebral Endpoints (ACTIVE) Trial (Miller et al. 2016). The phase 2 dosefinding study was a 24-week randomized, parallel group, double-blind, placebo-controlled study, comparing daily placebo; abaloparatide 20, 40 and $80 \mu \mathrm{g}$ and teriparatide $20 \mathrm{mg}$ in 222 women with postmenopausal osteoporosis (Leder et al. 2015). At 24 weeks, abaloparatide $80 \mu \mathrm{g}$ significantly increased BMD compared to placebo in the lumbar spine, femoral neck, and total hip $(P<0.05$ for all three comparisons). The direct comparison between abaloparatide and teriparatide yielded a more pronounced BMD increase at the total hip with abaloparatide $(2.6 \%$ vs $0.5 \%)$, while no significant differences were noted at the lumbar spine and femoral neck. The ACTIVE trial was an 18-month phase 3, double-blinded, randomized clinical trial, comparing daily injections of $80 \mu \mathrm{g}$ abaloparatide with placebo, and open-label $20 \mu \mathrm{g}$ teriparatide in 2463 women with postmenopausal osteoporosis (Miller et al. 2016) (Table 1). When compared to placebo, abaloparatide led to a greater increase in BMD at the lumbar spine (11.2 vs $0.6 \%, P<0.01$ ), at the femoral neck (3.6 vs $-0.4 \%$, $P<0.01)$, and at the total hip $(2.6 \%$ vs $-0.1 \%, P<0.01)$. Compared to teriparatide, abaloparatide led to more pronounced improvements in percentage BMD at the total hip ( 4.2 vs $3.3 \%, P<0.01)$, and at the femoral neck (3.6 vs $2.7 \%, P<0.01$ ).

Primary endpoint of this trial was the reduction of vertebral fractures with abaloparatide compared to placebo. Vertebral fractures were reduced by $86 \%$ (HR: 0.14, 95\% CI: 0.05-0.39, $P<0.001)$. Compared to placebo, non-vertebral fractures were significantly reduced in the abaloparatide (HR: 0.57, 95\% CI: 0.32-1.00, $P<0.049$ ) but not in the teriparatide group (HR: $0.79,95 \%$ CI: 0.43-1.45, 
Table 1 Published phase 3 trials assessing abaloparatide or romosozumab for the treatment of postmenopausal osteoporosis.

\begin{tabular}{l}
\hline Trial \\
\hline Abaloparatide \\
ACTIVE \\
Romosozumab \\
FRAME \\
ARCH \\
\hline
\end{tabular}

\begin{tabular}{c}
\hline $\mathbf{N C T}$ \\
\hline 01343004 \\
01575834 \\
01631214 \\
\hline
\end{tabular}

\begin{tabular}{c}
\hline $\boldsymbol{n}$ \\
\hline 2463 \\
7180 \\
4093 \\
\hline
\end{tabular}

\begin{tabular}{c}
\hline Duration (months) \\
\hline 18 \\
$12 / 12^{*}$ \\
24 \\
\hline
\end{tabular}

\begin{tabular}{c}
\hline LS BMD (vs placebo) \\
\hline+11.2 vs $+0.6 \%$ \\
+13.3 vs $+0.0 \% \#$ \\
+15.2 vs $+7.1 \% \S$ \\
\hline
\end{tabular}

\begin{tabular}{c}
\hline Vertebral Fx (vs placebo) \\
\hline$-86 \%$ (RR: $0.14 ; 95 \% \mathrm{Cl}: 0.05-0.39 ; P<0.011)$ \\
$-73 \%$ (RR: $0.27 ; 95 \% \mathrm{Cl}: 0.16-0.47 ; P<0.001)^{\#}$ \\
$-48 \%$ (RR: $0.52 ; 95 \% \mathrm{Cl}: 0.40-0.66 ; P<0.001)^{\S}$ \\
\hline
\end{tabular}

*12 months romosozumab vs placebo, followed by 12 months denosumab for both groups; \#at 12 months; ${ }^{5}$ compared to alendronate.

Fx, fracture; LS BMD, lumbar spine bone mineral density; $n$, number of subjects included; NCT, ClinicalTrials.gov registry number.

$P=0.44)$. Of note, this trial was not powered to directly compare the effect of abaloparatide and teriparatide on vertebral fractures and the definition of non-vertebral fractures chosen for analysis was different from previous teriparatide studies, which had demonstrated efficacy with regards to non-vertebral events. Exploratory analyses of major osteoporotic fractures revealed a favorable outcome for abaloparatide vs teriparatide (HR: 0.45, 95\% CI: $0.21-0.95, P=0.03)$.

Hypercalcemia was more frequent with teriparatide (6.1\%) and abaloparatide (3.4\%), than with placebo $(0.4 \%)$, with a trend for higher hypercalcemia occurrence under teriparatide when compared to abaloparatide $(P=0.06)$. Other adverse effects were similar between the groups and comprised gastrointestinal complaints, injection-site reactions, dizziness and myalgia. Regarding neoplasms, there were no remarkable trends between groups. Reports of tumorigenicity are based on studies in rats. In a study comparing abaloparatide with teriparatide and placebo over 2 years, a higher incidence of osteosarcoma and osteoblastoma was noted with abaloparatide and teriparatide when compared to placebo (Jolette et al. 2017). Of note, doses used were estimated to result in 10-25-fold higher exposure than the usual doses prescribed in humans. The Osteosarcoma Surveillance Study detected no association between teriparatide and subsequent osteosarcoma in humans after 7 years of follow-up (Andrews et al. 2012).

A post hoc analysis of the ACTIVE trial underscored abaloparatide's anti-fracture efficacy across a wide variety of ages and baseline risks, independently of the presence or absence of previous fractures (Cosman et al. 2017a). These results were confirmed in another post hoc analysis of the ACTIVE trial which used the FRAX tool to assess baseline fracture probability (McCloskey et al. 2017). Furthermore, a sub-study of the ACTIVE trial in 105 women who underwent bone biopsies reported normal cell morphology in all three groups through histological analysis, while histomorphometry revealed lower eroded surface with abaloparatide vs placebo $(P<0.05)$, and higher bone porosity with both drugs vs placebo $(P<0.05)$, implying that both drugs induce bone remodeling (Moreira et al. 2017).

The ACTIVExtend study was designed to evaluate the effects of sequential treatment with alendronate in 1139 patients who were treated either with abaloparatide or with placebo in the original ACTIVE trial. A 6-month interim analysis published showed a significant increase in BMD in the abaloparatide/alendronate group compared to the placebo/alendronate group at all skeletal sites $(+12.8$, +5.5 and $+4.5 \%$ vs $+3.5,+1.4$ and $+0.5 \%$, for lumbar spine, total hip and femoral neck respectively, $P<0.001$ for all comparisons between the two groups). There was an $87 \%$ relative risk reduction for new morphometric vertebral fractures with abaloparatide/alendronate $(P<0.001$ compared to placebo/alendronate), and a $52 \%$ risk reduction in the incidence of non-vertebral fractures with abaloparatide/alendronate group $(P=0.02$ compared to placebo/alendronate) (Cosman et al. 2017b). Final results from ACTIVExtend confirmed an $84 \%$ relative risk reduction in new vertebral fractures in the abaloparatide/ alendronate compared to placebo/alendronate after 43 months of cumulative treatment (Bone et al. 2018).

A randomized, double-blind, placebo-controlled, phase 3 study to investigate the efficacy of abaloparatide in the treatment of male osteoporosis is currently in progress (NCT03512262). In addition, the application of abaloparatide as a dermal patch is also being evaluated and a pivotal trial is expected to commence in 2019.

\section{Sclerostin in bone}

Over the last 15 years, the genetic etiology of many bone diseases has been unraveled. Major findings led to a better understanding of the regulation of bone mass through the Wnt canonical signaling pathway, which was subsequently established as the major regulator of osteoblast function (Gaur et al. 2005) (Fig. 2). Wnt signaling also induces osteoprotegerin (OPG), an osteoblast secreted factor that negatively regulates osteoclast formation, highlighting 
A

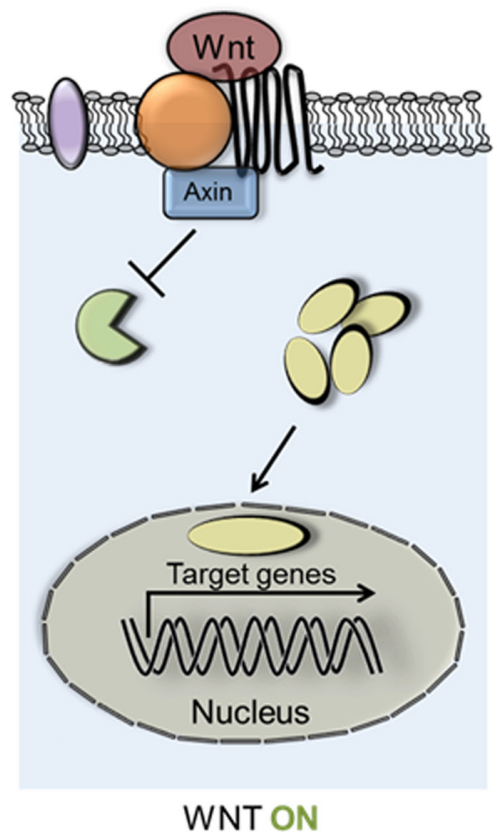

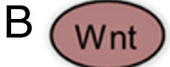
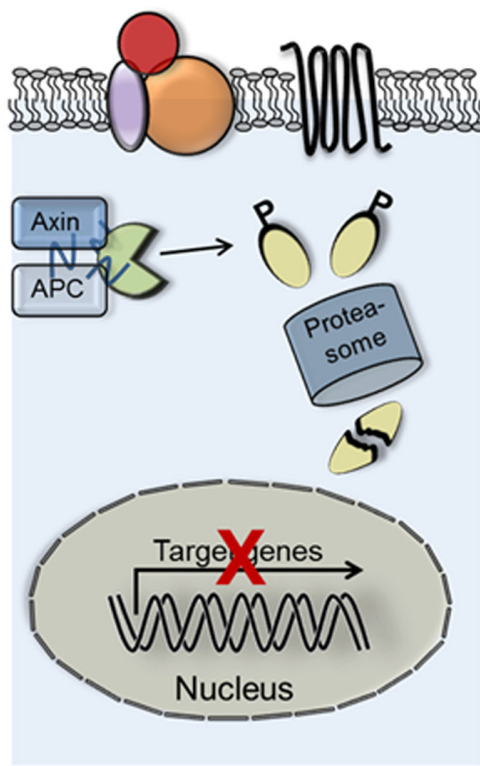

WNT OFF

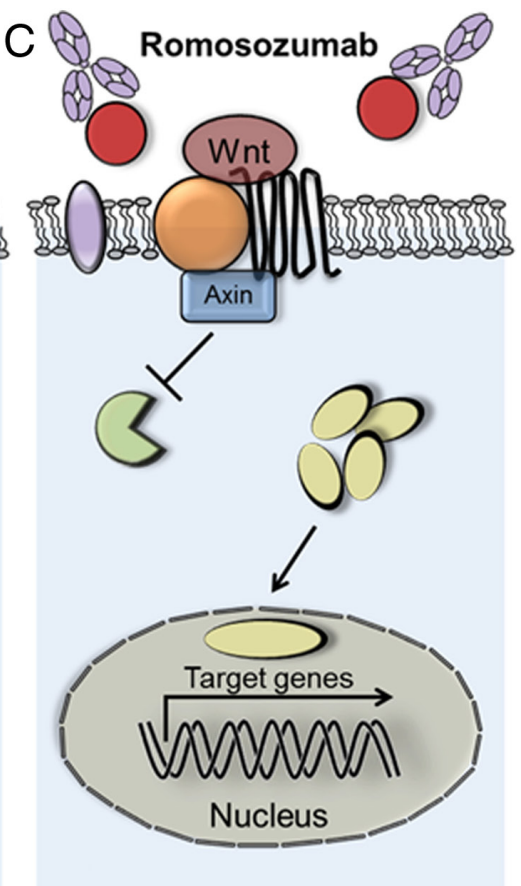

WNT ON

Figure 2

Romosozumab is a monoclonal antibody directed against the Wnt inhibitor sclerostin. Canonical Wnt signaling can be activated by binding of Wnt ligands to a receptor complex comprising of the low-density lipoprotein receptor-related proteins (LRP) 5 or 6 and a protein member of the frizzled (Fzd) family. Fzd mediates the recruitment of Axin, thereby forming a complex which inhibits $\beta$-catenin phosphorylation by glycogen synthase kinase 3 (GSK-3 $\beta$ ). Free $\beta$-catenin accumulates in the cytoplasm, translocates to the nucleus and activates target gene expression (A). Wnt inhibitors like sclerostin bind LRP5/6, recruit Kremen and prevent binding to the Fzd receptor as well as induce internalization of LRP5/6. This stabilizes a $\beta$-catenin destruction complex consisting of Axin, GSK-3 $\beta$, and adenomatous polyposis-coli protein (APC). Phosphorylated $\beta$-catenin is polyubiquitinylated and degraded by the proteasome which leads to a disruption of Wnt target gene expression (B). The monoclonal antibody romosozumab neutralizes sclerostin thereby restoring Wnt signaling (C).

the complexity of this pathway on bone homeostasis (Lerner \& Ohlsson 2015). Initially, mutations of the gene coding for the low-density lipoprotein receptor-related protein 5 (LRP5) receptor, a key component of the Wnt signaling pathway, were identified as the cause of the osteoporosis-pseudoglioma syndrome (OMIM \# 259770) (Gong et al. 2001), and syndromes of inherited high bone mass (Boyden et al. 2002, Little et al. 2002). Further studies elucidated the role of sclerostin as a major inhibitor of the Wnt signaling pathway through binding to the LRP5/6 co-receptors (Li et al. 2005). In addition to its action to abrogate the canonical Wnt signaling pathway, sclerostin has also been shown to enhance osteoclastogenesis by stimulating the production of the receptor activator of NF-kB ligand (RANKL) from osteocytes (Wijenayaka et al. 2011).

Two inherited human diseases presenting with a high bone mass phenotype, sclerosteosis (OMIM \# 269500) (Balemans et al. 2001) and van Buchem's disease (Balemans et al. 2002, Staehling-Hampton et al. 2002)
(OMIM \# 239100), were found to be caused by inactivating mutations of SOST, the gene encoding sclerostin. Individuals with sclerosteosis have a high BMD and are resistant to fractures, but display symptoms of bone overgrowth, such as cranial nerve compression (Gardner et al. 2005). Van Buchem's disease typically causes a similar clinical phenotype as sclerosteosis, including high bone mass and elevated levels of bone formation markers (Wergedal et al. 2003). The role of sclerostin as a pivotal genetic determinant of BMD and fracture risk was further highlighted by evidence deriving from genome-wide association studies (Estrada et al. 2012). Following these findings, monoclonal antibodies binding sclerostin were developed as possible anabolic agents.

\section{Sclerostin inhibition}

The therapeutic potential of antibodies directed against sclerostin was first evaluated in preclinical studies. The administration of an anti-sclerostin antibody 
over 5 weeks in ovariectomized rats led to pronounced increases of bone mass and strength at several skeletal sites ( $\mathrm{Li}$ et al. 2009). These effects were reproduced in ovariectomized cynos treated with two once-monthly injections of an anti-sclerostin antibody (Ominsky et al. 2010). Another study in male cynos highlighted the potential of anti-sclerostin antibodies to improve trabecular microarchitecture (Matheny et al. 2017). These animal studies have contributed to the elucidation of antisclerostin antibodies' mechanism of action. A marked, albeit transient, increase in bone formation on previously quiescent bone surfaces is followed by a decrease in bone resorption, thus uncoupling the two phases of bone metabolism (Ominsky et al. 2017).

\section{Romosozumab}

Romosozumab is a fully humanized monoclonal IgG2 antibody with high specificity for human sclerostin. In a phase I multidose study in 72 healthy men and postmenopausal women, a single dose of romosozumab was associated with dose-related increases of bone formation markers up to $200 \%$ above baseline and reduction of bone resorption markers of up to $50 \%$ below baseline, while the highest dose achieved BMD increases of 4-5\% at the lumbar spine (Padhi et al. 2011). These results were confirmed by a second multidose phase I study, where romosozumab was administered over 3 months (Padhi et al. 2014). This study also included assessment of trabecular and cortical parameters via high-resolution QCT scans of the spine in 48 subjects and revealed significant increases in cortical BMD $(+9.5 \%, P<0.01)$ and cortical thickness $(+26.9 \%, P=0.06)$ as compared to placebo (Graeff et al. 2015). These improvements were maintained during the 3 months of follow-up.

In the phase 2 dose-finding study, 419 women with postmenopausal osteoporosis were randomized to receive either one of five romosozumab subcutaneous dosing regimens (ranging from $70 \mathrm{mg}$ every 3 months to $210 \mathrm{mg}$ every month), placebo, alendronate or teriparatide for 12 months (McClung et al. 2014). Treatment with romosozumab induced a rapid increase of bone formation markers, peaking at 1-3 months, which returned to baseline by month 6 and remained below baseline until the end of study. Conversely, there was a rapid decline in bone resorption markers, which remained suppressed until month 12. At 12 months, the average increases in BMD amounted to $11.3 \%$ at the lumbar spine, and $4.1 \%$ at the total hip, with the highest dose of romosozumab as compared to placebo (both $P<0.001$ ). These increases were more pronounced than those observed with teriparatide or alendronate. This study continued for one more year with a blinded protocol. During the second year BMD continued to increase at a slower rate, and both bone formation and resorption markers were suppressed, implying that romosozumab primarily acted as an antiresorptive during that period (McClung et al. 2018). At the end of year 2, eligible patients were re-randomized to either placebo or biannual injections of $60 \mathrm{mg}$ denosumab for 1 year. In those switched to placebo, BMD values decreased toward baseline, while in those switched to denosumab, BMD increased at the lumbar spine by $3.7 \%$ and at the total femur by $1.1 \%$, increases which were similar to the second year of romosozumab therapy (McClung et al. 2018).

The first phase III study to be published was the Fracture Study in Postmenopausal Women with Osteoporosis (FRAME) (Cosman et al. 2017c) (Fig. 1). The study recruited 7180 postmenopausal women with osteoporosis who were randomized to receive subcutaneous injections of either $210 \mathrm{mg}$ romosozumab once-monthly or placebo for 12 months, followed by an additional 12 months of denosumab $60 \mathrm{mg}$ every 6 months in both treatment arms. In the first 12 months, there was a $73 \%$ reduction for vertebral fractures $(P<0.001)$ and a 36\% reduction for clinical fractures $(P=0.008)$, while no statistically significant difference was seen for non-vertebral fractures. During the second year, a trend toward a benefit for nonvertebral and clinical fractures persisted in the former romosozumab group when compared to the former placebo group, although this did not reach statistical significance. Thus, this study established the efficacy of romosozumab to prevent vertebral fractures, while the prevention of nonvertebral fractures was not unequivocally underpinned by this study (Cosman et al. 2017c). In a recently published retrospective analysis of the data, the authors concluded that the 2-year BMD gains in the lumbar spine and hip with romosozumab approximated the effect of 7 years of continuous denosumab administration (Cosman et al. 2018).

A second phase III fracture endpoint trial $(\mathrm{ARCH})$ compared the effects of subcutaneous romosozumab $210 \mathrm{mg}$ once-monthly with oral alendronate $70 \mathrm{mg}$ once weekly for 12 months, followed by another year where both treatment groups received alendronate. Over a period of 24 months, a $48 \%$ lower risk of new vertebral fractures was observed in the romosozumab-to-alendronate group $(P<0.001)$. Furthermore the study reported a $27 \%$ lower risk for clinical fractures with romosozumab $(P<0.001)$, while non-vertebral fractures were reduced by $19 \%$ $(P=0.04)$, and hip fractures were reduced by $38 \%(P=0.02)$ 
by romosozumab (Saag et al. 2017), although neither of the phase III studies was powered to evaluate the effect of romosozumab therapy on hip fracture risk.

In a study evaluating the respective efficacy of romosozumab and teriparatide in patients formerly treated with bisphosphonates, the mean percentage change from baseline in total hip areal BMD was 2.6\% (95\% CI: $2.2-3.0$ ) in the romosozumab group and $-0.6 \%$ $(-1.0$ to -0.2$)$ in the teriparatide group, highlighting the effects of transitioning to different osteoanabolic agents in patients pre-treated with bisphosphonates (Langdahl et al. 2017).

In the FRAME study, mild injection-site reactions were the most common adverse effect observed in $5.2 \%$ of patients treated with romosozumab vs $2.9 \%$ of patients treated with placebo. Other adverse events, including instances of hyperostosis, cardiovascular events, osteoarthritis and cancer, were balanced between the groups. One atypical femoral fracture and two cases of osteonecrosis of the jaw were observed in the romosozumab group (Cosman et al. 2017c).

In the ARCH study, a numerical imbalance in serious cardiovascular events was described with more events in the romosozumab arm than with alendronate ( 2.5 vs 1.9\%; OR: 1.31 ; 95\% CI: 0.85-2.00), during the first double-blind period of 12 months (Saag et al. 2017). Possible explanations could lie in a cardio-protective effect of alendronate or the difference in the study populations between the two phase III studies with subjects of the ARCH study displaying a higher cardiovascular risk at baseline (Khosla 2017). Based on these results, the FDA has required additional data prior to deciding on the approval of romosozumab for the treatment of postmenopausal osteoporosis.

\section{Additional sclerostin antibody trials}

Additional sclerostin antibodies are in clinical development and have been tested in early clinical trials. BPS804 is a fully human, neutralizing monoclonal antibody directed against sclerostin. BPS804 has been investigated in patients with hypophosphatasia. Eight patients were treated with three ascending doses of BPS804 and followed for 16 weeks after the last dose. Treatment was well generally well tolerated and transient increases in bone formation markers as well as transient decreases in bone resorption markers were seen. At the end of the study, BMD at the lumbar spine had increased by 3.9\% (Seefried et al. 2017). In another open-label, phase2a trial BPS804 was tested in nine patients with moderate osteogenesis imperfecta and referenced to five control patients. At day 43, all measured markers of bone formation were significantly increased to baseline, whereas CTX was suppressed by $44 \%$ compared to baseline. Lumber BMD was increased by $4 \%$ at day 141 $(P=0.038)$ (Glorieux et al. 2017).

Blosozumab is another sclerostin antibody that has been tested in a phase 2 trial (Recker et al. 2015). In this dose-ranging study increasing subcutaneous doses of blosozumab ( $180 \mathrm{mg}$ every 4 weeks, $180 \mathrm{mg}$ every 2 weeks, $270 \mathrm{mg}$ every 2 weeks or placebo) were given. Dosedependent increases in BMD were seen in spine, femoral neck and total hip BMD as compared with placebo.

\section{Summary}

There is currently a clear unmet need for additional bone anabolic agents for the treatment of osteoporosis. A better understanding of the cellular events and signaling pathways mediating osteoblast differentiation and function have contributed to the development of novel osteoanabolic agents. With romosozumab and abaloparatide having successfully completed phase 3 trials in postmenopausal osteoporosis, these agents are in the process of obtaining approval or, in the case of abaloparatide, have already been approved by the FDA. All presented trials (FRAME, ARCH, ACTIVE) have transitioned into an antiresorptive sequence therapeutic regimen, and there is a general consent that an antiresorptive therapy is required to maintain and stabilize bone improvements gained under anabolic therapy. The increase in treatment options will certainly benefit patients. However, choosing the right drug in the right sequence is becoming more complex and requires a more detailed knowledge about their mode of action. In summary, current efficacy data of the new drugs appear convincing, and the utilization of these agents followed by a potent antiresorptive drug will probably ameliorate existing therapeutic options for severe osteoporosis. Further studies will help better define the safety profiles and long-term effects of these agents, as well as their efficacy in settings other than postmenopausal osteoporosis.

\section{Declaration of interest}

Dr Rachner reports honoraria for lectures from Amgen and Roche, advisory boards from Merck and educational grants from UCB. Dr Hofbauer reports reports honoraria for lectures and educational material from Alexion, Amgen, Merck, Roche and Shire to his institution and himself, and grant support from Amgen and Novartis to his institution. Dr Göbel has nothing to declare. Dr Tsourdi reports honoraria for lectures from Amgen and educational fees from UCB. 


\section{Funding}

This work was supported by the Schwerpunktprogramm-2084 $\mu$ Bone of Deutsche Forschunggemeinschaft to $T D R, L C H$, and $A G$ and the Frauenhabilitationsstipendium of TU Dresden to ET.

\section{References}

Andrews EB, Gilsenan AW, Midkiff K, Sherrill B, Wu Y, Mann BH \& Masica D 2012 The US postmarketing surveillance study of adult osteosarcoma and teriparatide: study design and findings from the first 7 years. Journal of Bone and Mineral Research 27 2429-2437. (https://doi.org/10.1002/jbmr.1768)

Balemans W, Ebeling M, Patel N, Van Hul E, Olson P, Dioszegi M, Lacza C, Wuyts W, Van Den Ende J, Willems P, et al. 2001 Increased bone density in sclerosteosis is due to the deficiency of a novel secreted protein (SOST). Human Molecular Genetics 10 537-543. (https://doi.org/10.1093/hmg/10.5.537)

Balemans W, Patel N, Ebeling M, Van Hul E, Wuyts W, Lacza C, Dioszegi M, Dikkers FG, Hildering P, Willems PJ, et al. 2002 Identification of a $52 \mathrm{~kb}$ deletion downstream of the SOST gene in patients with van Buchem disease. Journal of Medical Genetics 39 91-97. (https://doi.org/10.1136/jmg.39.2.91)

Bone HG, Wagman RB, Brandi ML, Brown JP, Chapurlat R, Cummings SR, Czerwiński E, Fahrleitner-Pammer A, Kendler DL, Lippuner K, et al. 201710 years of denosumab treatment in postmenopausal women with osteoporosis: results from the phase 3 randomised FREEDOM Trial and open-label extension. Lancet Diabetes Endocrinology 5 513-523. (https://doi.org/10.1016/S22138587(17)30138-9)

Bone HG, Cosman F, Miller PD, Williams GC, Hattersley G, Hu MY, Fitzpatrick LA, Mitlak B, Papapoulos S, Rizzoli R, et al. 2018 ACTIVExtend: 24 months of alendronate after 18 months of abaloparatide or placebo for postmenopausal osteoporosis. Journal of Clinical Endocrinology and Metabolism 103 2949-2957. (https://doi. org/10.1210/jc.2018-00163)

Boyce EG, Mai Y \& Pham C 2018 Abaloparatide: review of a nextgeneration parathyroid hormone agonist. Annals of Pharmacotherapy 52 462-472. (https://doi.org/10.1177/1060028017748649)

Boyden LM, Mao JH, Belsky J, Mitzner L, Farhi A, Mitnick MA, Wu D, Insogna K \& Lifton RP 2002 High bone density due to a mutation in LDL-receptor-related protein 5. New England Journal of Medicine 346 1513-1521. (https://doi.org/10.1056/NEJMoa013444)

Cheloha RW, Gellman SH, Vilardaga JP \& Gardella TJ 2015 PTH receptor-1 signalling-mechanistic insights and therapeutic prospects. Nature Reviews Endocrinology 11 712-24. (https://doi.org/10.1038/ nrendo.2015.139)

Cosman F, Hattersley G, Hu MY, Williams GC, Fitzpatrick LA \& Black DM 2017a Effects of Abaloparatide-SC on fractures and bone mineral density in subgroups of postmenopausal women with osteoporosis and varying baseline risk factors. Journal of Bone and Mineral Research 32 17-23. (https://doi.org/10.1002/ jbmr.2991)

Cosman F, Miller PD, Williams GC, Hattersley G, Hu MY, Valter I, Fitzpatrick LA, Riis BJ, Christiansen C, Bilezikian JP, et al. 2017 b Eighteen months of treatment with subcutaneous abaloparatide followed by 6 months of treatment with alendronate in postmenopausal women with osteoporosis: results of the ACTIVExtend trial. Mayo Clinic Proceedings 92 200-210. (https://doi. org/10.1016/j.mayocp.2016.10.009)

Cosman F, Crittenden DB, Adachi JD, Binkley N, Czerwinski E, Ferrari S, Hofbauer LC, Lau E, Lewiecki EM, Miyauchi A, et al. 2017 c Romosozumab treatment in postmenopausal women with osteoporosis. New England Journal of Medicine 375 1532-1543. (https://doi.org/10.1056/NEJMoa1607948)
Cosman F, Crittenden DB, Ferrari S, Khan A, Lane NE, Lippuner K, Matsumoto T, Milmont CE, Libanati C \& Grauer A 2018 FRAME Study: the foundation effect of building bone with 1 year of Romosozumab leads to continued lower fracture risk after transition to denosumab. Journal of Bone and Mineral Research 33 1219-1226. (https://doi.org/10.1002/jbmr.3427)

Doyle N, Varela A, Haile S, Guldberg R, Kostenuik PJ, Ominsky MS, Smith SY \& Hattersley G 2018 Abaloparatide, a novel PTH receptor agonist, increased bone mass and strength in ovariectomized cynomolgus monkeys by increasing bone formation without increasing bone resorption. Osteoporosis International 29 685-697. (https://doi.org/10.1007/s00198-017-4323-6)

Estrada K, Styrkarsdottir U, Evangelou E, Hsu YH, Duncan EL, Ntzani EE, Oei L, Albagha OM, Amin N, Kemp JP, et al. 2012 Genome-wide meta-analysis identifies 56 bone mineral density loci and reveals 14 loci associated with risk of fracture. Nature Genetics 44 491-501. (https://doi.org/10.1038/ng.2249)

Frolik CA, Black EC, Cain RL, Satterwhite JH, Brown-Augsburger PL, Sato M \& Hock JM 2003 Anabolic and catabolic bone effects of human parathyroid hormone (1-34) are predicted by duration of hormone exposure. Bone 33 372-379.

Gardner JC, van Bezooijen RL, Mervis B, Hamdy NA, Löwik CW, Hamersma H, Beighton P \& Papapoulos SE 2005 Bone mineral density in sclerosteosis; affected individuals and gene carriers. Journal of Clinical Endocrinology and Metabolism 90 6392-6395. (https://doi. org/10.1210/jc.2005-1235)

Gaur T, Lengner CJ, Hovhannisyan H, Bhat RA, Bodine PV, Komm BS, Javed A, van Wijnen AJ, Stein JL, Stein GS, et al. 2005 Canonical WNT signaling promotes osteogenesis by directly stimulating Runx 2 gene expression. Journal of Biological Chemistry 280 33132-33140. (https://doi.org/10.1074/jbc.M500608200)

Gensure RC, Gardella TJ \& Jüppner H 2005 Parathyroid hormone and parathyroid hormone-related peptide, and their receptors. Biochemical and Biophysical Research Communications 328 666-678. (https://doi.org/10.1016/j.bbrc.2004.11.069)

Glorieux FH, Devogelaer JP, Durigova M, Goemaere S, Hemsley S, Jakob F, Junker U, Ruckle J, Seefried L \& Winkle PJ 2017 BPS804 anti-sclerostin antibody in adults with moderate osteogenesis imperfecta: results of a randomized phase $2 \mathrm{a}$ trial. Journal of Bone and Mineral Research 32 1496-1504. (https://doi.org/10.1002/jbmr.3143)

Glover SJ, Eastell R, McCloskey EV, Rogers A, Garnero P, Lowery J, Belleli R, Wright TM \& John MR 2009 Rapid and robust response of biochemical markers of bone formation to teriparatide therapy. Bone 45 1053-1058. (https://doi.org/10.1016/j.bone.2009.07.091)

Gong YQ, Slee RB, Fukai N, Rawadi G, Roman-Roman S, Reginato AM, Wang H, Cundy T, Glorieux FH, Lev D, et al. 2001 LDL receptorrelated protein 5 (LRP5) affects bone accrual and eye development. Cell 107 513-523. (https://doi.org/10.1016/S0092-8674(01)00571-2)

Graeff C, Campbell GM, Peña J, Borggrefe J, Padhi D, Kaufman A, Chang S, Libanati C \& Glüer CC 2015 Administration of Romosozumab improves vertebral trabecular and cortical bone as assessed with quantitative computed tomography and finite element analysis. Bone 81 364-369. (https://doi.org/10.1016/j. bone.2015.07.036)

Hattersley G, Dean T, Corbin BA, Bahar H \& Gardella TJ 2016 Binding selectivity of abaloparatide for PTH-Type-1-receptor conformations and effects on downstream signaling. Endocrinology 157 141-149. (https://doi.org/10.1210/en.2015-1726)

Horwitz MJ, Tedesco MB, Garcia-Ocaña A, Sereika SM, Prebehala L, Bisello A, Hollis BW, Gundberg CM \& Stewart AF 2010 Parathyroid hormone-related protein for the treatment of postmenopausal osteoporosis: defining the maximal tolerable dose. Journal of Clinical Endocrinology and Metabolism 95 1279-1287. (https://doi. org/10.1210/jc.2009-0233)

Jolette J, Attalla B, Varela A, Long GG, Mellal N, Trimm S, Smith SY, Ominsky MS \& Hattersley G 2017 Comparing the incidence of bone 
tumors in rats chronically exposed to the selective PTH type 1 receptor agonist abaloparatide or PTH(1-34). Regulatory Toxicology and Pharmacology 86 356-365. (https://doi.org/10.1016/j. yrtph.2017.04.001)

Khosla S 2017 Romosozumab-on track or derailed? Nature Reviews Endocrinology 13 697-698. (https://doi.org/10.1038/nrendo.2017.136)

Langdahl BL, Libanati C, Crittenden DB, Bolognese MA, Brown JP, Daizadeh NS, Dokoupilova E, Engelke K, Finkelstein JS, Genant HK, et al. 2017 Romosozumab (sclerostin monoclonal antibody) versus teriparatide in postmenopausal women with osteoporosis transitioning from oral bisphosphonate therapy: a randomised, open-label, phase 3 trial. Lancet 390 1585-1594. (https://doi. org/10.1016/S0140-6736(17)31613-6)

Leder BZ 2017 Parathyroid hormone and parathyroid hormone-related protein analogs in osteoporosis therapy. Current Osteoporosis Reports 15 110-119. (https://doi.org/10.1007/s11914-017-0353-4)

Leder BZ, O'Dea LS, Zanchetta JR, Kumar P, Banks K, McKay K, Lyttle CR \& Hattersley G 2015 Effects of abaloparatide, a human parathyroid hormone-related peptide analog, on bone mineral density in postmenopausal women with osteoporosis. Journal of Clinical Endocrinology and Metabolism 100 697-706. (https://doi.org/10.1210/ jc.2014-3718)

Legrand J, Becret A, Fisch C, Attia M, De Jouffrey S, Dong JZ, Woon CW, Claude CJ \& Cullen MD 2001 BIM-44058, a novel PTHrP analog, increases bone formation but not bone resorption histomorphometric parameters in old ovariectomized cynomolgus monkeys. Journal of Bone and Mineral Research 16 S539.

Lerner UH \& Ohlsson C 2015 The WNT system: background and its role in bone. Journal of Internal Medicine 277 630-649. (https://doi. org/10.1111/joim.12368)

Lewiecki EM \& Miller PD 2013 Skeletal effects of primary hyperparathyroidism: bone mineral density and fracture risk. Journal of Clinical Densitometry 16 28-32. (https://doi.org/10.1016/j. jocd.2012.11.013)

Li X, Zhang Y, Kang H, Liu W, Liu P, Zhang J, Harris SE \& Wu D 2005 Sclerostin binds to LRP5/6 and antagonizes canonical Wnt signaling. Journal of Biological Chemistry 280 19883-19887. (https://doi. org/10.1074/jbc.M413274200)

Li XD, Ominsky MS, Warmington KS, Morony S, Gong J, Cao J, Gao Y, Shalhoub V, Tipton B, Haldankar R, et al. 2009 Sclerostin antibody treatment increases bone formation, bone mass, and bone strength in a rat model of postmenopausal osteoporosis. Journal of Bone and Mineral Research 24 578-588. (https://doi.org/10.1359/jbmr.081206)

Little RD, Carulli JP, Del Mastro RG, Dupuis J, Osborne M, Folz C, Manning SP, Swain PM, Zhao SC, Eustace B, et al. 2002 A mutation in the LDL receptor-related protein 5 gene results in the autosomal dominant high-bone-mass trait. American Journal of Human Genetics 70 11-19. (https://doi.org/10.1086/338450)

Matheny JB, Torres AM, Ominsky MS \& Hernandez CJ 2017 Romosozumab treatment converts trabecular rods into trabecular plates in male cynomolgus monkeys. Calcified Tissue International 101 82-91. (https://doi.org/10.1007/s00223-017-0258-3)

McCloskey EV, Johansson H, Oden A, Harvey NC, Jiang H, Modin S, Fitzpatrick L \& Kanis JA 2017 The effect of abaloparatide-sc on fracture risk is independent of baseline FRAX fracture probability: a post hoc analysis of the ACTIVE study. Journal of Bone and Mineral Research 32 1625-1631. (https://doi.org/10.1002/jbmr.3163)

McClung MR, Grauer A, Boonen S, Bolognese MA, Brown JP, DiezPerez A, Langdahl BL, Reginster JY, Zanchetta JR, Wasserman SM, et al. 2014 Romosozumab in postmenopausal women with low bone mineral density. New England Journal of Medicine 370 412-420. (https://doi.org/10.1056/NEJMoa1305224)

McClung MR, Brown JP, Diez-Perez A, Resch H, Caminis J, Meisner P, Bolognese MA, Goemaere S, Bone HG, Zanchetta JR, et al. 2018 Effects of 24 months of treatment with Romosozumab followed by 12 months of denosumab or placebo in postmenopausal women with low bone mineral density: a randomized, double-blind, phase 2, parallel group study. Journal of Bone and Mineral Research 33 1397-1406. (https://doi.org/10.1002/jbmr.3452)

McCudden CR, Hains MD, Kimple RJ, Siderovski DP \& Willard FS 2005 G-protein signaling: back to the future. Cellular and Molecular Life Sciences 62 551-577. (https://doi.org/10.1007/s00018-004-4462-3)

Miller PD, Hattersley G, Riis BJ, Williams GC, Lau E, Russo LA, Alexandersen P, Zerbini CA, Hu MY, Harris AG, et al. 2016 Effect of abaloparatide vs placebo on new vertebral fractures in postmenopausal women with osteoporosis: a randomized clinical trial. JAMA 316 722-733. (https://doi.org/10.1001/jama.2016.11136)

Moreira CA, Fitzpatrick LA, Wang Y \& Recker RR 2017 Effects of abaloparatide-sc (BA058) on bone histology and histomorphometry: the ACTIVE phase 3 trial. Bone 97 314-319. (https://doi. org/10.1016/j.bone.2016.11.004)

Ominsky MS, Vlasseros F, Jolette J, Smith SY, Stouch B, Doellgast G, Gong J, Gao Y, Cao J, Graham K, et al. 2010 Two doses of sclerostin antibody in cynomolgus monkeys increases bone formation, bone mineral density, and bone strength. Journal of Bone and Mineral Research 25 948-959. (https://doi.org/10.1002/jbmr.14)

Ominsky MS, Boyce RW, Li X \& Ke HZ 2017 Effects of sclerostin antibodies in animal models of osteoporosis. Bone 96 63-75. (https://doi.org/10.1016/j.bone.2016.10.019)

Padhi D, Jang G, Stouch B, Fang L \& Posvar E 2011 Single-dose, placebocontrolled, randomized study of AMG 785, a sclerostin monoclonal antibody. Journal of Bone and Mineral Research 26 19-26. (https://doi. org/10.1002/jbmr.173)

Padhi D, Allison M, Kivitz AJ, Gutierrez MJ, Stouch B, Wang C \& Jang G 2014 Multiple doses of sclerostin antibody Romosozumab in healthy men and postmenopausal women with low bone mass: a randomized, double-blind, placebo-controlled study. Journal of Clinical Pharmacology 54 168-178. (https://doi.org/10.1002/ jcph.239)

Papapoulos SE 2011 Use of bisphosphonates in the management of postmenopausal osteoporosis. Annals of the New York Academy of Sciences 1218 15-32. (https://doi. org/10.1111/j.1749-6632.2010.05767.x)

Recker RR, Benson CT, Matsumoto T, Bolognese MA, Robins DA, Alam J, Chiang AY, Hu L, Krege JH, Sowa H, et al. 2015 A randomized, double-blind phase 2 clinical trial of Blosozumab, a sclerostin antibody, in postmenopausal women with low bone mineral density. Journal of Bone and Mineral Research 30 216-24. (https://doi. org/10.1002/jbmr.2351)

Saag KG, Petersen J, Brandi ML, Karaplis AC, Lorentzon M, Thomas T, Maddox J, Fan M, Meisner PD \& Grauer A 2017 Romosozumab or alendronate for fracture prevention in women with osteoporosis. New England Journal of Medicine 377 1417-1427. (https://doi. org/10.1056/NEJMoa1708322)

Seefried L, Baumann J, Hemsley S, Hofmann C, Kunstmann E, Kiese B, Huang Y, Chivers S, Valentin MA, Borah B, et al. 2017 Efficacy of anti-sclerostin monoclonal antibody BPS804 in adult patients with hypophosphatasia. Journal of Clinical Investigation 127 2148-2158. (https://doi.org/10.1172/JCI83731)

Smith SY, Jolette J \& Turner CH 2009 Skeletal health: primate model of postmenopausal osteoporosis. American Journal of Primatology $\mathbf{7 1}$ 752-765. (https://doi.org/10.1002/ajp.20715)

Staehling-Hampton K, Proll S, Paeper BW, Zhao L, Charmley P, Brown A, Gardner JC, Galas D, Schatzman RC, Beighton P, et al. 2002 A 52-kb deletion in the SOST-MEOX1 intergenic region on 17q12-q21 is associated with van Buchem disease in the Dutch population. American Journal of Medical Genetics 110 144-152. (https://doi. org/10.1002/ajmg.10401)

Toulis KA, Anastasilakis AD, Polyzos SA \& Makras P 2011 Targeting the osteoblast: approved and experimental anabolic agents for the treatment of osteoporosis. Hormones 10 174-195. (https://doi. org/10.14310/horm.2002.1308) 
Varela A, Chouinard L, Lesage E, Smith SY \& Hattersley G 2017a One year of abaloparatide, a selective activator of the PTH1 receptor, increased bone formation and bone mass in osteopenic ovariectomized rats without increasing bone resorption. Journal of Bone and Mineral Research 32 24-33. (https://doi.org/10.1002/jbmr.3003)

Varela A, Chouinard L, Lesage E, Guldberg R, Smith SY, Kostenuik PJ \& Hattersley G 2017b One year of abaloparatide, a selective peptide activator of the PTH1 receptor, increased bone mass and strength in ovariectomized rats. Bone 95 143-150. (https://doi.org/10.1016/j. bone.2016.11.027)

Wergedal JE, Veskovic K, Hellan M, Nyght C, Balemans W, Libanati C, Vanhoenacker FM, Tan J, Baylink DJ \& Van Hul W 2003 Patients with Van Buchem disease, an osteosclerotic genetic disease, have elevated bone formation markers, higher bone density, and greater derived polar moment of inertia than normal. Journal of Clinical Metabolism and Endocrinology 88 5778-5783. (https://doi. org/10.1210/jc.2003-030201)

Wijenayaka AR, Kogawa M, Lim HP, Bonewald LF, Findlay DM \& Atkins GJ 2011 Sclerostin stimulates osteocyte support of osteoclast activity by a RANKL-dependent pathway. PLOS ONE 6 e25900. (https://doi.org/10.1371/journal.pone.0025900)

Wysolmerski JJ 2012 Parathyroid hormone-related protein: an update. Journal of Clinical Endocrinology and Metabolism 97 2947-2956. (https://doi.org/10.1210/jc.2012-2142)

Received in final form 5 September 2018

Accepted 12 September 2018

Accepted Preprint published online 12 September 2018
(C) 2019 Society for Endocrinology Published by Bioscientifica Ltd. Printed in Great Britain 